\title{
GLTGS 晶体生长及其热释电性能
}

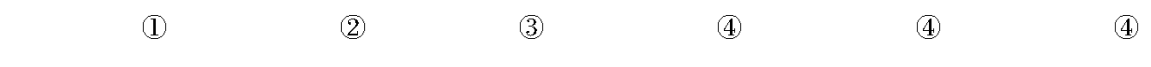

(1)北京工业大学材料科学与工程学院, 北京 100022; (2)国家建材局人工晶体研究所, 北京 100018; (3山东大学晶体材料研究 所, 济南 250100; (4)北京工业大学环境与能源工程学院, 北京 100022)

摘要 根据结构与性能的关系, 选择胍基乙酸作为掺质, 采用水溶液降温法生长了 GLTGS 晶体, 发现掺质的存在使晶体的生长形态、单胞参数和解理性均发生了变化. 对所生长晶体的热释电性 能进行了系统测量. 结果表明, 掺质显著地增强了晶体的热释电效应, 与纯 TGS 晶体相比, GLTGS 晶体在 $20 \sim 30^{\circ} \mathrm{C}$ 的温度范围内, 热释电系数增加了 $49.3 \% ~ 55.8 \%$, 品质因子增加了 35.4\% 49.3\%. 另外, 经过测量晶体的电滞回线发现, 在相同条件下, GLTGS 晶体的矫顽电场比纯 TGS 晶体约增大一倍,

关键词 GLTGS 晶体生长 热释电系数 品质因子 矫顾电场

硫酸三甘氨酸 $\left[\left(\mathrm{NH}_{2} \mathrm{CH}_{2} \mathrm{COOH}\right)_{3} \cdot \mathrm{H}_{2} \mathrm{SO}_{4}\right]$ (简称 $\mathrm{TGS}$ )晶体是 1956 年发现的一种铁电晶 体 ${ }^{[1]}$. 到目前为止, 它是一种应用最广泛的水溶性热释电晶体材料, 大量用于红外探测、红外 摄像等高技术领域 ${ }^{[2,3]}$. 但由于该晶体居里点偏低 $\left(49.2^{\circ} \mathrm{C}\right)$ 和易退极化等缺点, 在某种程度上限 制了其应用. 几十年来, 人们对 TGS 晶体的改性做了大量工作, 取得的成果主要体现在以下 几方面: 気化可将其居里点提高到 $62^{\circ} \mathrm{C}^{[4]}$; 加入 $\mathrm{L}-\alpha$-丙氨酸可锁定极化 ${ }^{[5 \sim 7]}$; 加入磷酸 ${ }^{[8,9]}$ 、砷 酸 ${ }^{[6]}$ 或其他掺质 ${ }^{[10 ~ 12]}$ 可提高其热释电性能等. 为了得到更佳效果, 人们仍然在不断地进行研究 和探索.

考虑到胍基乙酸 $\left[\mathrm{H}_{2} \mathrm{~N}(\mathrm{NH}) \mathrm{CNHCH}_{2} \mathrm{COOH}\right]$ 的结构与甘氨酸有较大的相似性，分子的极性 也较大, 应该容易进入 TGS 晶体, 期望以其极性来锁定极化或提高晶体的热释电性能. 因而 我们生长了掺胍基乙酸的 TGS(简称 GLTGS)晶体, 并进行了相应的热释电性能测试, 结果是 令人满意的.

\section{1 材料与方法}

( i ) GLTGS 晶体生长溶液的配制. 以甘氨酸 $\left(\mathrm{NH}_{2} \mathrm{CH}_{2} \mathrm{COOH}\right)$ (B.R.) 水溶液与硫酸 $\left(\mathrm{H}_{2} \mathrm{SO}_{4}\right)(\mathrm{A} . \mathrm{R}$.) 水溶液 $(1: 1)$ 通过下列反应形成 TGS:

$$
3 \mathrm{NH}_{2} \mathrm{CH}_{2} \mathrm{COOH}+\mathrm{H}_{2} \mathrm{SO}_{4} \rightarrow\left(\mathrm{NH}_{2} \mathrm{CH}_{2} \mathrm{COOH}\right)_{3} \cdot \mathrm{H}_{2} \mathrm{SO}_{4}
$$

若溶液带淡黄色, 需以活性炭脱色, 得到无色透明溶液. 必要时重结晶 1 或 2 次, 然后配 制成一定饱和点的 TGS 溶液. 再往溶液中加入胍基乙酸(B.R.), 使其在溶液中的浓度达 $10 \%$, 即溶液中胍基乙酸摩尔数/(胍基乙酸摩尔数+氨基乙酸摩尔数 $) \times 100 \%=10 \%$ ，从而制得 GLTGS 晶体生长溶液.

（ii）晶体生长方法与条件. 生长 GLTGS 晶体所采用的方法为水溶液缓慢降温法, 生长 条件为: 选用 $\perp b$ 向、尺寸为 $a=8 \mathrm{~mm}, b=1 \mathrm{~mm}, c=14 \mathrm{~mm}$ 的片状 TGS 籽晶, 溶液体积 $270 \mathrm{~mL}$, 掺质浓度 $10 \%, \mathrm{pH}=2.0$, 生长温度区间为 $46 \sim 40^{\circ} \mathrm{C}$, 控温精度 $\pm 0.02^{\circ} \mathrm{C}$.

(iii) 晶体单胞参数与热释电性能测试方法. 采用 $\mathrm{X}$ 射线单晶衍射仪测定晶体的单胞参 数. 采用电荷积分法测定晶体的热释电系数, 采用 HP4274A 型 LRC 测量仪测定晶体的介电 
常数 $\varepsilon_{\mathrm{r}}$, 采用 TRC-1 型准静态电滞回线测量仪测量晶体的电滞回线. 将晶体加工成厚度为 1 $\mathrm{mm}$, 边长为 $6 \mathrm{~mm}$ 的正方形晶片, 晶片厚度方向平行于 $b$ 向, 即晶片大面垂直于 $b$ 向, 在其 两个大面上镀金电极后得测试样品, 该样品经极化处理后进行各种性质测试.

\section{2 实验}

\section{1 晶体生长形态}

按上节的方法和条件生长出了数块 GLTGS 晶体, 其中之一的 $c$ 向俯视图如图 1(a)所示, 其他几块晶体与之类似, 图 1(b)为与之对照的纯 TGS 晶体. 由图 1 可见, GLTGS 晶体的外形 与纯 TGS 相比, 总的来看变化不大, 但有些晶面如 $\{212\},\{203\},\{011\}$ 以及 $\{001\}$ 等面族的大 小和形态均发生了变化, 这些晶面不再是严格的平面, 而是微有凸起或有小晶面出现, 并使原 来的晶棱也不规则了, 同时又出现了一些额外的不规则晶棱, 如图 1(a)中的 1,2,3,4 等. 这些不规 则晶棱与晶面的出现也无明显规律性, 即不同块晶体出现不规则晶面和晶棱的数目和位置均 不相同. 但这类面和棱的不规则性均出现在晶体的 $\{212\},\{203\},\{011\},\{001\}$ 面族区域, 在这 些区域的晶体内部并无宏观缺陷, 而[001]晶带所属各晶面(平行于 $c$ 向的柱面, 即图中边缘各 直线所示晶面)和[101] 晶带所属各晶面(如 $(\overline{1} \overline{1} 1),(\overline{1} 11),(\overline{1} 21),(\overline{1} \overline{2} 1)$ 等晶面)也未见明显变 化. 另外在晶体样品的加工过程中发现, GLTGS 晶体的(010)解理性比纯 TGS 晶体明显减弱.

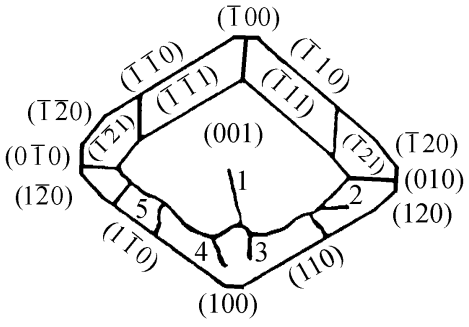

(a)

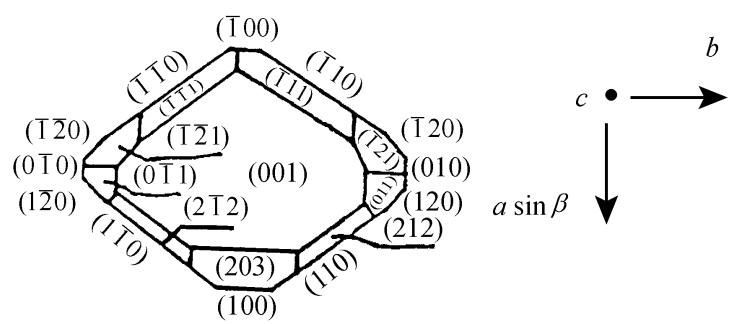

(b)

图 1 GLTGS(a)和 TGS(b)晶体 $c$ 向俯视图

2.2 晶体单胞参数的测量

采用 $X$ 射线单晶衍射仪精确测定了 GLTGS 晶体的单胞参数, 并在同样条件下测定了纯 TGS 晶体的单胞参数, 结果列于表 1 .

表 1 GLTGS 与 TGS 晶体的单胞参数

\begin{tabular}{cccccccc}
\hline 单胞参数 & $a_{0} / \mathrm{nm}$ & $b_{0} / \mathrm{nm}$ & $c_{0} / \mathrm{nm}$ & $\alpha /\left(^{\circ}\right)$ & $\beta /\left(^{\circ}\right)$ & $\gamma /\left({ }^{\circ}\right)$ & $V / \mathrm{nm}^{3}$ \\
\hline TGS & 0.57268 & 1.26454 & 0.91696 & 89.964 & 105.534 & 90.067 & 0.63978 \\
GLTGS & 0.57325 & 1.26400 & 0.91623 & 90.045 & 105.539 & 90.012 & 0.63963 \\
\hline
\end{tabular}

可见, 与纯 TGS 相比, GLTGS 晶体的 $a_{0}$ 有所增大, 而 $b_{0}$ 和 $c_{0}$ 则有所减小, 总的结果其单 胞体积比 TGS 晶体的略有减小.

\section{3 晶体热释电性能}

分别测定了 GLTGS 晶体的热释电系数 $p$ 随温度 $T$ 的变化曲线、介电常数 ${ }_{\mathrm{r}}$ 与温度 $T$ 的 关系曲线以及不同温度下的电滞回线, 如图 2 4 所示, 其中图 4 为 GLTGS 晶体在 $27.8^{\circ} \mathrm{C}$ 时 的电滞回线. 
由图 2 4 可见, GLTGS 晶体的热释电系数明显地高于纯 TGS 晶体, 介电常数也比纯 TGS 的高, 随温度的变化趋势与纯 TGS 相似. 与纯 TGS 一样, GLTGS 晶体的内偏压场 $E_{\mathrm{b}}=0$, 但 矫顽电场 $E_{\mathrm{c}}$ 约为纯 TGS 的 2 倍.

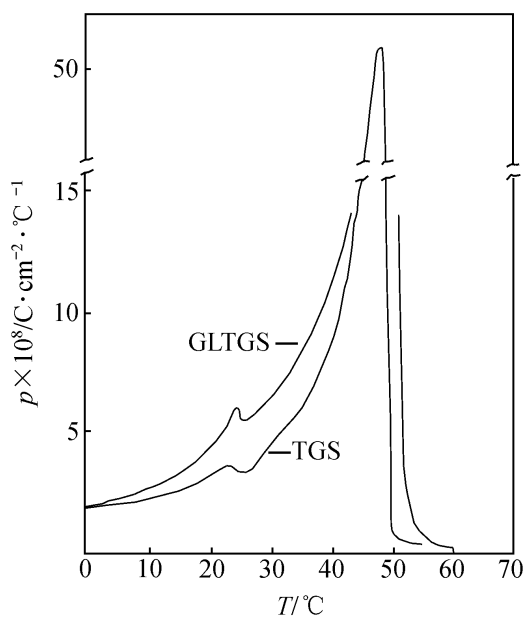

图 2 GLTGS 和 TGS 晶体的热释电系数与 温度的关系

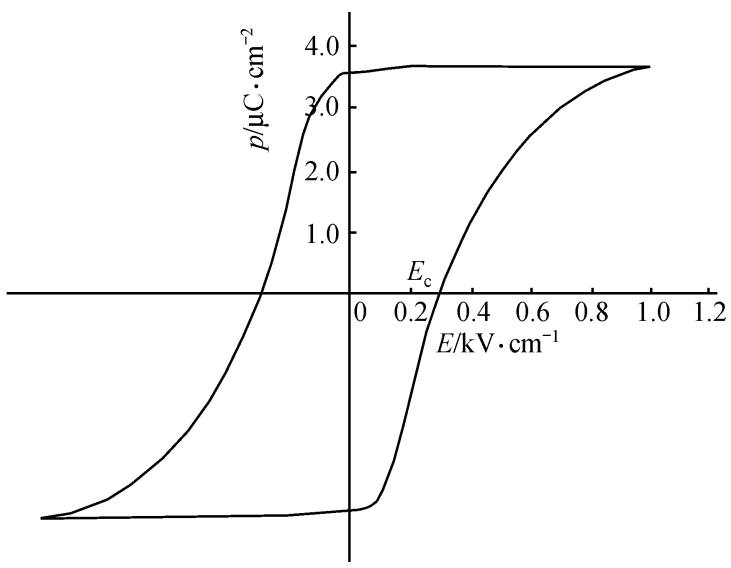

(a)

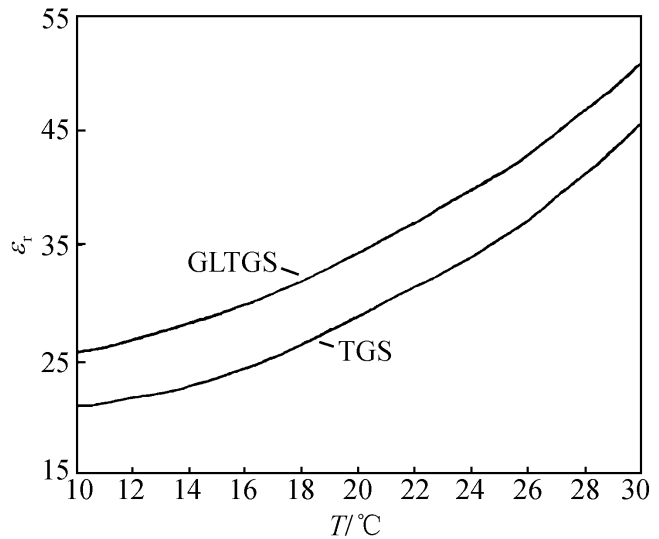

图 3 GLTGS 和 TGS 晶体介电常数与温度的关系

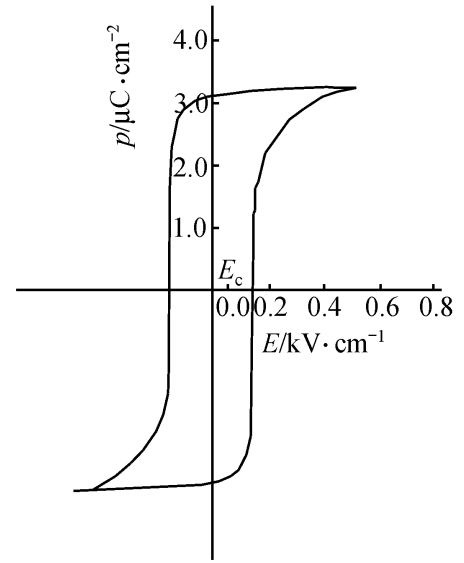

(b)

图 4 GLTGS(a)和 TGS(b)晶体的电滞回线

为了更清楚地展现出 GLTGS 晶体的热释电性能, 现将 $20^{\circ} \mathrm{C}$ 和 $30^{\circ} \mathrm{C}$ 时该晶体的性能测试 结果列于表 2, 并与纯 TGS 晶体进行对比.

表 2 GLTGS 和 TGS 晶体的热释电性能

\begin{tabular}{|c|c|c|c|c|}
\hline \multirow{2}{*}{ 参数 } & \multicolumn{2}{|c|}{$20^{\circ} \mathrm{C}$} & \multicolumn{2}{|c|}{$30^{\circ} \mathrm{C}$} \\
\hline & GLTGS & TGS & GLTGS & TGS \\
\hline$p \times 10^{8} / \mathrm{C} \cdot \mathrm{cm}^{-2} \cdot{ }^{9} \mathrm{C}^{-1}$ & 4.48 & 3.00 & 6.70 & 4.30 \\
\hline$\varepsilon_{\mathrm{r}}$ & 32 & 29 & 48 & 46 \\
\hline$M \times 10^{10} / \mathrm{C} \cdot \mathrm{cm}^{-2} \cdot{ }^{9} \mathrm{C}^{-1}$ & 14.00 & 10.34 & 13.96 & 9.35 \\
\hline
\end{tabular}




\section{3 讨论}

\section{1 掺质胍基乙酸对晶体结构、解理性与晶体形态的影响}

当晶体中掺入胍基乙酸后，由于胍基乙酸中的胍基与甘氨酸中的氨基相比，增加了一个 $\mathrm{C}$ 原子、两个 $\mathrm{N}$ 原子和两个 $\mathrm{H}$ 原子, 这样不但增大了原来所占体积, 而且由于比甘氨酸增加 了两个 $\mathrm{N}-\mathrm{H}$ 键导致形成更多的氢键, 在一定程度上改变了晶体的内部结构, 加强了 $b$ 向的 键合能力 (这一点也可以从 $b_{0}$ 的减小反映出来), 从而使 GLTGS 晶体(010)向解理性能减弱. 同 时由于结构的变化引起晶体形态发生变化.

\section{2 胍基乙酸对 $\mathrm{TGS}$ 晶体的热释电性能的影响}

品质因子 $M\left(=p / \varepsilon_{\mathrm{r}}\right)$ 是衡量晶体热释电性能优劣的主要指标之一, $M$ 越大, 性能越好. 通过 测量晶体不同温度下的热释电系数 $p$ 和介电常数 $\varepsilon_{\mathrm{r}}$ 经计算得出相应温度下的品质因子 $M$ (见 表 2). 发现在 $20 \sim 30^{\circ} \mathrm{C}$ 范围内(即室温下)GLTGS 晶体的 $p$ 值比纯 TGS 晶体增加了 49.3\% 55.8\%， 尽管其 $\varepsilon_{\mathrm{r}}$ 同时有所增加，而 $M$ 值也比纯 TGS 提高了 $35.4 \% ~ 49.3 \%$. 在目前众多的 TGS 掺质 中, 能够这么大幅度提高其热释电性能是不多见的, 这与掺质分子胍基乙酸进入晶体后加强 了 $b$ 向 (即晶体的极轴方向)的键合能力密切相关. 胍基的存在增加了氢键的数目, 继而增加了 正电荷重心偏离分子平面的程度, 也就增加了晶体极轴方向的偶极矩, 从而使晶体的自发极 化强度、热释电系数和介电常数均有不同程度的提高. 总的结果是较大幅度地提高了晶体的 品质因子. 另外, $\mathrm{TGS}$ 晶体中掺入胍基乙酸后, 其矫项电场 $E_{\mathrm{c}}$ 增大了约一倍. 这也是一个好现 象, 虽然没有像掺入 L- $\alpha$-丙氨酸那样有较强的内偏压场而锁定极化, 但这对晶体的退极化起 到一定的阻碍作用. 这也与胍基乙酸本身有较强的极性和能形成较多氢键以及分子体积相对 较大等因素有关, 这些因素使其偶极矩难以翻转, 晶体中的电畴转向从而变得困难了.

\section{3 胍基乙酸掺入晶体的可能性分析}

从以下几个方面我们认为胍基乙酸已掺入 TGS 晶体：(1) 胍基乙酸与甘氨酸分子结构相 似，只是胍基与氨基的差别(胍基中含有氨基，这两个基团也有相似的性质)，而分子其余部分 则完全相同, 因此容易掺入; (2) GLTGS 晶体形态、晶胞参数和解理性等与纯 TGS 晶体相比 均发生了明显的变化, 数次生长的 GLTGS 晶体均出现了类似现象, 而我们多次生长纯 TGS 晶体时从未出现该现象，因此可以判断，这种现象确为胍基乙酸的掺入所致；(3) 更重要的是, GLTGS 晶体的热释电性能与纯 TGS 晶体相比有大幅度提高, 重复测试结果表明这不是偶然 的测量误差. 由此认定胍基乙酸已掺入晶体，并影响了晶体的形态、结构和性能.

另外我们曾试图采用红外和紫外光谱法取得胍基乙酸掺入晶体的直接证据，但发现胍基 乙酸和甘氨酸的硫酸盐红外光谱谱带重叠, 紫外光谱也有类似情况, 难以区分, 又由于样品为 硫酸盐难以气化, 不能采用气相色谱-质谱联用技术. 目前尚未找到适当的方法直接定性或定 量地测量 GLTGS 晶体中胍基乙酸的含量.

\section{4 结论}

（1）采用水溶液降温法可以生长出内部无宏观缺陷的优质 GLTGS 晶体. 晶体形态与纯 TGS 晶体相比, 出现了一些不规则的晶面和晶棱, 且晶体的(010)面解理性降低了.

(2) GLTGS 晶体的单胞参数与纯 TGS 相比, $a_{0}$ 略有增大, 而 $b_{0}$ 和 $c_{0}$ 略有减小, 晶胞体积 
有所减小.

(3) 与纯 TGS 相比, 室温下 GLTGS 晶体的热释电系数增加了 $49.3 \%$ 55\%, 品质因子提 高了 $35.4 \% \sim 49.3 \%$.

（4） TGS 晶体中掺入胍基乙酸后, 其矫顽电场增大了一倍左右, 这样对晶体的退极化现象 起到一定的阻碍作用, 能够提高晶体的使用周期.

(5) 由以上 4 个结论可以判断, 胍基乙酸已掺入晶体.

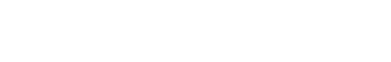

1 Matthias B T, Miller G E, Remeika J P. Ferroelectricity of glycine sulfate. Phys Rev, 1956, 104: 849 850

2 张克从. 近代晶体学基础(上). 北京: 科学出版社, 1998. 251 254

3 王希敏, 常新安, 张克从. TGS 系列晶体生长与性质的研究. 人工晶体学报, 1991, 20(1): 95 102

4 Beerman H P. Characterization of fully deuterated triglycine sulfate $\left(\mathrm{ND}_{2} \mathrm{CD}_{2} \mathrm{COOD}\right)_{3} \mathrm{D}_{2} \mathrm{SO}_{4}$. Ferroelectrics, 1974, 8: $653 \sim 656$

5 Bye K L, Whipps P W, Keve E T. High internal bias fields in TGS(L-alanine). Ferroelectrics, 1972, 4: $253 \sim 256$

6 房昌水, 王 民, 张克从. 一种新的热释电晶体一ATGSAs 的生长和性质. 科学通报, 1985, 30(8): 580 582

7 Nakatani N. Ferroelectric domain structure and internal bias field in DL- $\alpha$-alanine doped tryglycine sulfate. Japanese Journal of Applied Physics, 1991, 30(12A): 3445 3449

8 Ravi G, Anbukumar S, Ramasamy P. Growth and characterization of triglycine sulpho-phosphate mixed crystals. Materials Chemistry and Physics, 1994, 37: 180 183

9 Arunmozhi G, Jayavel R, Subramanian C. Ferroelectric studies on amino acids mixed TGSP single crystals. Materials Letters, 1998, 33: 251 254

10 王希敏, 常新安, 张克从, 等. 新型热释电材料——HTGS 晶体生长与性能. 科学通报, 1992, 37(21): 2007 2010

11 张克从, 常新安, 王希敏. 掺质 TGS 系列晶体的生长形态与表征的研究. 人工晶体学报, 1997, 26(2): 95 101

12 Aravazhi S, Jayavel R, Subramanian C. Growth and characterization of benzophenone and urea doped triglycine sulphate crystals. Ferroelectrics, 1997, 200: 279 286

(1999-10-10 收稿, 2000-05-10 收修改稿) 